\title{
UPON A CONVERGENCE RESULT IN THE THEORY OF THE PADÉ TABLE
}

\author{
BY \\ P. WYNN
}

Abstract. The main theorem of this paper is the following: Let $M_{v}, b_{v}(\nu=1,2, \ldots$, $n)$ be two sets of finite positive real numbers, with $b_{1}>b_{2}>\cdots>b_{n}$, and let $\sigma(s)$ be a bounded nondecreasing function for $a \leqq s \leqq b$ where $0 \leqq a \leqq b<b_{n}$; denote the Padé quotients derived from the series expansion of the function

$$
f(z)=\sum_{v=1}^{n} \frac{M_{v}}{\left(1+b_{v} z\right)}+\int_{a}^{b} \frac{d \sigma(s)}{1+z s}
$$

in ascending powers of $z$ by $\left\{R_{i, j}(z)\right\}$; let $\mathfrak{D}$ be the open disc $|z|<b^{-1}$ cut along the real segment $\left(-b^{-1},-b_{1}^{-1}\right]$; define a progressive sequence of Padé quotients to be one in which the successor $R_{i^{\prime \prime}, j^{\prime \prime}}(z)$ to $R_{i^{\prime}, j^{\prime}}(z)$ is such that either $i^{\prime \prime}>i^{\prime}$ and $j^{\prime \prime} \geqq j^{\prime}$ or $i^{\prime \prime} \geqq i^{\prime}$ and $j^{\prime \prime}>j^{\prime}$; then any infinite progressive sequence of quotients $\left\{R_{i, j}(z)\right\}$ for which $i \geqq n$ and $j \geqq n$ converges uniformly for $z \in \mathfrak{T}$ to $f(z)$.

The proof proceeds in a number of stages; we first consider those progressive sequences bounded by the main diagonal sequence $R_{r, r}(z)(r=n, n+1, \ldots)$ and the row sequence $R_{n, n+r}(z)(r=0,1, \ldots)$. It follows from a result of Markoff that all diagonal sequences of the form $R_{n+r, n+n^{\prime}+r}(z)$, where $n^{\prime}$ is a finite nonnegative integer and $r=0,1, \ldots$, converge uniformly for $z \in \mathfrak{D}$ to $f(z)$. From a theorem of de Montessus de Ballore the row sequence $R_{n, n+r}(z)(r=0,1, \ldots)$ converges uniformly for $z \in \mathfrak{D}$ to $f(z)$. From a result of the author the backward diagonal sequences $R_{n+r, 2 m-n-r}(z)$ $(r=0,1, \ldots, m-n)$ and $R_{n+r, 2 m-n-r+1}(z)(r=0,1, \ldots, m-n+1)$, where $m$ is a finite positive integer, are, when $z$ is real and positive, respectively monotonically decreasing and monotonically increasing. Hence the result of the theorem is true for the restricted progressive sequences in question when $z$ is real and positive. Using the result of de Montessus de Ballore, and extending a result of Nevanlinna to the theory of the Pade table in question, it is shown that there exists a finite positive integer $r^{\prime}$ such that all quotients $R_{n+r, n+r^{\prime \prime}+r}\left(r=0,1, \ldots ; r^{\prime \prime}=r^{\prime}, r^{\prime}+1, \ldots\right)$ are uniformly bounded for $z \in \mathfrak{D}^{\prime}$, where $\mathfrak{D}^{\prime}$ is that part of $\mathfrak{D}$ from which points lying in the neighborhood of the negative real axis have been excluded. Thus, using the StieltjesVitali theorem, all progressive sequences of Padé quotients taken from the latter double array converge uniformly for $z \in \mathfrak{D}^{\prime}$ to $f(z)$. That the diagonal sequences of the complementary set $R_{n+r, n+r^{\prime \prime}+r}(z)\left(r=0,1, \ldots ; r^{\prime \prime}=0,1, \ldots, r^{\prime}-1\right)$ each converge uniformly for $z \in \mathfrak{D}^{\prime}$ to $f(z)$ follows from Markoff's result. Hence the result of the theorem is true for the restricted progressive sequences when $z \in \mathfrak{D}^{\prime}$; that this result also holds for values of $z \in \mathfrak{D}$ lying in the neighborhood of the negative real axis (and not, therefore, belonging to $\mathfrak{D}^{\prime}$ ) is proved by the use of a theorem of Tschebyscheff. The Padé quotients lying below the principal diagonal can be associated with a function $\hat{f}(z)$ having many of the properties of $f(z)$, and the proof outlined above may be extended to the progressive sequences bounded by the principal diagonal and the column sequence $R_{n+r, n}(z)(r=0,1, \ldots)$. The two partial results are then combined.

Received by the editors October 26, 1970 and, in revised form, May 21, 1971.

AMS 1970 subject classifications. Primary 30A22; Secondary 40A15. 
The main theorem. In this paper we prove the following result:

THEOREM. Let $M_{v}, b_{v}(\nu=1,2, \ldots, n)$ be two sets of $n(1 \leqq n<\infty)$ finite positive real numbers, with $b_{1}>b_{2}>\cdots>b_{n}$, and let $\sigma(s)$ be a bounded nondecreasing real valued function for $a \leqq s \leqq b$, where $0 \leqq a<b<b_{n}$; denote the Padé quotients derived from the series expansion of the function

$$
f(z)=\sum_{v=1}^{n} \frac{M_{v}}{1+b_{v} z}+\int_{a}^{b} \frac{d \sigma(s)}{1+z s}
$$

in ascending powers of $z$ by $\left\{R_{i, j}(z)\right\}$; let $\mathfrak{D}$ be the open disc $|z|<b^{-1}$ cut along the real segment $\left(-b^{-1},-b_{1}^{-1}\right]$; define a progressive sequence of Padé quotients to be one in which the successor $R_{i^{\prime \prime}, j^{\prime \prime}}(z)$ to $R_{i^{\prime}, j^{\prime}}(z)$ is such that either $i^{\prime \prime} \geqq i^{\prime}$ and $j^{\prime \prime}>j^{\prime}$, or $i^{\prime \prime}>i^{\prime}$ and $j^{\prime \prime} \geqq j^{\prime} ;$ then any infinite progressive sequence of quotients $\left\{R_{i, j}(z)\right\}$ for which $i \geqq n$ and $j \geqq n$ converges uniformly to $f(z)$ in $\mathfrak{D}$.

Some theory. Since the above is a rather deep result, we preface its proof by some definitions and lemmata. (For the theories of the Padé table and of continued fractions derived from power series, the reader is referred to the standard works of Perron [1] and Wall [2].)

We are concerned with rational functions of the form

$$
R_{i, j}(z)=\left(\sum_{\nu=0}^{\tilde{j}} \vartheta_{\nu}^{(i, j)} z^{\nu}\right) /\left(\sum_{\nu=0}^{\tilde{i}} \kappa_{\nu}^{(i, j)} z^{\nu}\right) \quad(i \geqq 0, j \geqq 0)
$$

derived from the power series

$$
f(z)=\sum_{v=0}^{\infty} t_{v} z^{v} \quad\left(t_{0} \neq 0\right) .
$$

The coefficients $\left\{\kappa_{v}^{(i, j)}\right\},\left\{\vartheta_{v}^{(i, j)}\right\}$ are obtained from those of $f(z)$ by solving the set of $i+j+1$ equations

$$
\begin{array}{rlrl}
\tilde{\kappa}_{v}^{(i, j)}=0 \quad(\nu>i), \quad \sum_{\nu=0}^{r} \tilde{\kappa}_{v}^{(i, j)} t_{r-v} & =0 \quad(r=j+1, j+2, \ldots, j+i+1) \\
& =\tilde{\vartheta}_{r}^{(i, j)} & (r=0,1, \ldots, j)
\end{array}
$$

for $\tilde{\kappa}_{v}^{(i, j)}(\nu=0,1, \ldots, i)$ (not all zero) $\tilde{\vartheta}_{v}^{(i, j)}(\nu=0,1, \ldots, j)$, and reducing the quotient

$$
\left(\sum_{\nu=0}^{j} \tilde{\vartheta}_{\nu}^{(i, j)} z^{\nu}\right) /\left(\sum_{\nu=0}^{i} \tilde{\kappa}_{v}^{(i, j)} z^{v}\right)
$$

to its irreducible form (2) in which $\kappa_{0}^{(i, j)}=1$. The series expansion of $R_{i, j}(z)$ in ascending powers of $z$ agrees with $f(z)$ to a certain number of terms; in particular, if (4) is itself irreducible, such agreement holds at least for the terms $t_{v} z^{v}(\nu=0$, $1, \ldots, i+j) . R_{i, j}(z)$ is the Padé quotient [3] of order $(i, j)$ derived from $f(z)$. The formal derivation of the rational function $R_{i, j}(z)$ was first carried out by Jacobi [4]; the theory of its derivation was placed upon a rigorous basis by Frobenius [5]; a systematic study of the functions $\left\{R_{i, j}(z)\right\}$, with particular reference to their significance in the theory of continued fractions, was carried out by Padé [3]. 
The quotients $R_{i, j}(z)(i=0,1, \ldots ; j=0,1, \ldots)$ may be set in a two dimensional array in which $i$ denotes a row number and $j$ a column number; this is the Padé table. We shall append to the Padé table the quotient $R_{0,-1}(z)=0$. The quotients $R_{r, r}(z)(r=0,1, \ldots)$ lie upon the principal diagonal of the Padé table. We shall call the diagonal upon which the quotients $R_{r, r-1}(z)(r=0,1, \ldots)$ lie the subprincipal diagonal.

We are principally concerned with Stieltjes functions [6], i.e. functions having the form

$$
g(z)=\int_{a^{\prime}}^{b^{\prime}} \frac{d \tilde{\sigma}(s)}{1+z s} \quad(0 \leqq a<b \leqq \infty)
$$

where $\tilde{\sigma}(s)$ is a bounded nondecreasing real valued function for $a \leqq s \leqq b$ such that the integral expressions

$$
t_{v}=(-1)^{v} \int_{a^{\prime}}^{b^{\prime}} s^{v} d \tilde{\sigma}(s) \quad(\nu=0,1, \ldots)
$$

are well defined. $g(z)$ is regular throughout the $z$-plane cut along the segment $\left[-a^{\prime-1},-b^{\prime-1}\right]$ of the negative real axis and, depending upon the nature of $\tilde{\sigma}(s)$, may also be defined at points belonging to this segment. The series $f(z)$ of formula (3) whose coefficients are given by expressions (6) represents $g(z)$ asymptotically for $-\pi<\arg (z)<\pi$; if $b^{\prime}<\infty, f(z)$ converges to $g(z)$ for $|z|<b^{\prime-1}$.

LEMMA 1. The Padé table generated by the Stieltjes function (5) has the following properties:

(i) if $\tilde{\sigma}(s)$ is not a simple step function with a finite number of salti in the range $a^{\prime} \leqq s \leqq b^{\prime}$, each Padé quotient $R_{i, j}(z)$ has the irreducible form (2) in which $\tilde{\imath}=i, j=j$, $\kappa_{i}^{(i, j)} \neq 0, \vartheta_{0}^{(i, j)} \neq 0, \vartheta_{j}^{(i, j)} \neq 0$, and the quotients $\left\{R_{i, j}(z)\right\}$ are thus distinct;

(ii) if $\tilde{\sigma}(s)$ is a simple step function with salti of magnitude $\tilde{M}_{v}>0$ at the distinct points $s=s_{v}\left(a \leqq s_{v} \leqq b\right)\left(v=1,2, \ldots, n^{\prime}\right)$ and one (none) of the $\left\{s_{v}\right\}$ is zero, then all quotients $R_{i, j}(z)$ for which both $i \geqq n^{\prime}-1, j \geqq n^{\prime}-1\left(i \geqq n^{\prime}, j \geqq n^{\prime}-1\right)$ reduce to the rational function $g(z)$ of which (5) is then the integral representation; all other quotients have the irreducible form described above and are distinct.

For a fixed finite positive real value of $z$ the numerical values of the above Padé quotients lying upon and above the subprincipal diagonal form two diagonal meshes of nonincreasing and nondecreasing sequences: for a fixed finite value of $m \geqq 0$ both the forward diagonal sequence $R_{r, 2 m+r}(z)(r=0,1, \ldots)$ and the backward diagonal sequence $R_{r, 2 m-r}(z)(r=0,1, \ldots, m)$ are nonincreasing and have the value of $g(z)$ $(>0)$ as a lower bound, whilst both the forward diagonal sequence $R_{r, 2 m+r-1}(z)$ $(r=0,1, \ldots)$ and the backward diagonal sequence $R_{r, 2 m-r-1}(z)(r=0,1, \ldots, m)$ are nondecreasing and have the value of $g(z)$ as an upper bound; furthermore, distinct consecutive quotients of these sequences have distinct values.

Proof. The result of clause (i) is due to Van Vleck [7] (see [8] for an analysis of the convergence behavior of the Padé table in this case); clause (ii) is a trivial ex- 
tension of clause (i). The second main result of the lemma is due to the author [9] (a slightly more complicated result holds for the quotients lying beneath the principal diagonal).

The function $f(z)$ of formula (1) is a Stieltjes function: in the notation of formula (5), $\tilde{\sigma}(s)$ is the function obtained by setting $a^{\prime}=a, b^{\prime}=b_{1}, \tilde{\sigma}(s)=0(0 \leqq s<a)$; $\tilde{\sigma}(s)=\sigma(s)(a \leqq s \leqq b) ; \tilde{\sigma}(s)=\sigma(b)\left(b<s<b_{n}\right)$;

$$
\begin{array}{ll}
\tilde{\sigma}(s)=\sigma(b)+\sum_{v=r+1}^{n} M_{v} \quad & \left(b_{r+1} \leqq s<b_{r} ; r=n-1, n-2, \ldots, 1\right), \\
\tilde{\sigma}(s)=\sigma(b)+\sum_{v=1}^{n} M_{v} & \left(b_{1} \leqq s \leqq \infty\right) .
\end{array}
$$

LEMMA 2. Let $f(z)$ be the Stieltjes function of formula (1), and $\hat{f}(z)$ be the function defined by the relationship

$$
f(z)=t_{0} /(1+z \hat{f}(z))
$$

then $\hat{f}(z)$ has a representation of the form

$$
\sum_{v=1}^{n} \frac{\hat{M}_{v}}{1+\hat{b}_{v} z}+\int_{\hat{a}}^{\hat{b}} \frac{d \hat{\sigma}(s)}{1+z s}
$$

where $0<\hat{M}_{v}<\infty(\nu=1,2, \ldots, n), b_{v+1}<\hat{b}_{v}<b_{v}(\nu=1,2, \ldots, n-1), \hat{b}_{n}<b_{n}, \hat{b} \leqq b$, $\hat{a} \geqq a$, and $\hat{\sigma}(s)$ is a bounded nondecreasing function for $\hat{a} \leqq s \leqq \hat{b}$.

Denote the Padé quotients generated by $f(z)$ by $\left\{R_{i, j}(z)\right\}$ and those generated by $\hat{f}(z)$ by $\left\{\hat{R}_{i, j}(z)\right\} ;$ then

$$
R_{j+1, i}(z)=t_{0} /\left(1+z \hat{R}_{i, j}(z)\right) \quad(i=0,1, \ldots ; j=0,1, \ldots) .
$$

Proof. The first result was given in [9]. The second is a special case of a more general result holding for functions satisfying a relationship of the form (7), due to Padé [3].

LEMMA 3. Let $h(z)$, where $h(0) \neq 0$, be a function which is analytic in the circle $|z|=\xi$ except in the neighborhood of $n$ simple poles at the points $z=z_{v}(\nu=1,2, \ldots, n$ $<\infty)$ within this circle, and denote by $\left\{R_{i, j}(z)\right\}$ the Padé quotients generated by $h(z) ;$ then the sequence $R_{n, j}(z)(j=0,1, \ldots)$ converges uniformly to $h(z)$ in the punctured disc obtained by excluding the poles $\left\{z_{v}\right\}$ from the region $|z|<\xi$; if $h(z)$ has a singularity upon the circle $|z|=\xi$, the above sequence of Padé quotients diverges when $|z|>\xi$.

Proof. The above is a special case of a more general result relating to functions that are meromorphic in the neighborhood of the origin, due to de Montessus de Ballore [10], whose theory derives in great measure from Hadamard's thesis [11] on functions represented by their Taylor series expansions.

LEMMA 4. Let $r \geqq 1$ be a fixed finite integer. If $0<u_{v}<\infty(\nu=1,2, \ldots, r)$ then

$$
C_{r}(z)=\frac{u_{1}}{1+} \frac{u_{2} z}{1+} \cdots \frac{u_{r} z}{1}
$$


is a well determined irreducible rational function of the form

$C_{2 r^{\prime}}(z)=\left(\sum_{v=0}^{r^{\prime}} \vartheta_{v}^{\left(2 r^{\prime}\right)} z^{v}\right) /\left(\sum_{v=0}^{r^{\prime}} \kappa_{v}^{\left(2 r^{\prime}\right)} z^{\nu}\right) \quad\left(\kappa_{0}^{\left(2 r^{\prime}\right)}=1, \kappa_{r^{\prime}}^{\left(2 r^{\prime}\right)} \neq 0, \vartheta_{0}^{\left(2 r^{\prime}\right)} \neq 0, \vartheta_{r^{\prime}}^{\left(2 r^{\prime}\right)} \neq 0\right)$

if $r=2 r^{\prime}$, and of the form

$$
\begin{aligned}
C_{2 r^{\prime}+1}(z)=\left(\sum_{\nu=0}^{r^{\prime}+1} \vartheta_{\nu}^{\left(2 r^{\prime}+1\right)} z^{v}\right) /\left(\sum_{\nu=0}^{r^{\prime}} \kappa_{\nu}^{\left(2 r^{\prime}+1\right)} z^{v}\right) \\
\left(\kappa_{0}^{\left(2 r^{\prime}+1\right)}=1, \kappa_{r^{\prime}}^{\left(2 r^{\prime}+1\right)} \neq 0, \vartheta_{0}^{\left(2 r^{\prime}+1\right)} \neq 0, \vartheta_{r^{\prime}+1}^{\left(2 r^{\prime}+1\right)} \neq 0\right)
\end{aligned}
$$

if $r=2 r^{\prime}+1$. If the series $f(z)$ of formula (3) is generated by the Stieltjes function $g(z)$ of formula (5), then the continued fraction whose successive convergents have the form (9) with $r=1,2, \ldots$ may be derived from $f(z)$ by imposing the condition that the series expansion of $C_{r}(z)$ in ascending powers of $z$ should agree with $f(z)$ as far as the term $t_{r-1} z^{r-1}(r=1,2, \ldots)$. If $g(z)$ is a rational function, this continued fraction terminates with the convergent (of odd or even order depending upon the nature of $g(z)$ ) equivalent to $g(z)$; if $g(z)$ is not a rational function the continued fraction is nonterminating. In either case those of its coefficients that are defined satisfy the inequalities $0<u_{v}<\infty$.

Furthermore, the even order convergents that are defined may, in particular, be expressed in the form

$$
C_{2 r^{\prime}}(z)=\sum_{v=1}^{r^{\prime}} \frac{\mu_{v}^{\left(2 r^{\prime}\right)}}{1+\beta_{v}^{\left(2 r^{\prime}\right)} z}
$$

where $\mu_{\nu}^{\left(2 r^{\prime}\right)}>0, a^{\prime}<\beta_{v}^{\left(2 r^{\prime}\right)}<b^{\prime}\left(\nu=1,2, \ldots, r^{\prime}\right), \sum_{v=1}^{r^{\prime}} \mu_{\nu}^{\left(2 r^{\prime}\right)}=t_{0}$.

In the case in which the continued fraction is nonterminating and, in formula (5), $b^{\prime}<\infty$, the sequence of convergents $\left\{C_{r}(z)\right\}$ converges uniformly to $g(z)$ in any open domain of the z-plane lying within finite distance of the origin and not including any point of the real segment $\left(-a^{\prime-1}, b^{\prime-1}\right)$ as an interior point.

Proof. The first result concerning the nature of the function $C_{r}(z)$ is an elementary result in the theory of continued fractions with nonzero coefficients. A continued fraction derived from a power series as described in the theorem is said to correspond to the power series in question. The result concerning the existence of a corresponding continued fraction deriving from a Stieltjes function and the nature of its convergents is due to Tschebyscheff [12] and Stieltjes [3]. The last result, concerning convergence, is due to Markoff [13].

Lemma 5. Let $m \geqq 0$ be a fixed finite integer. Denote the Padé quotients generated by the Stieltjes function of formula $(5)$ by $\left\{R_{i, j}(z)\right\}$. Let $\left\{C_{r}^{(m)}(z)\right\}$ be the convergents of the corresponding continued fraction generated by the Stieltjes function

$$
g^{(m)}(z)=\int_{a^{\prime}}^{b^{\prime}} \frac{s^{m} d \tilde{\sigma}(s)}{1+z s},
$$


and set $C_{0}^{(m)}(z)=0$. Then, in the notation of formula (9),

$$
\begin{aligned}
R_{r, m+r-1}(z) & =\sum_{v=0}^{m-1} t_{v} z^{v}+z^{m} C_{2 r}^{(m)}(z), \\
R_{r, m+r}(z) & =\sum_{\nu=0}^{m-1} t_{v} z^{v}+z^{m} C_{2 r+1}^{(m)}(z)
\end{aligned}
$$

for values of $r$ for which the convergents $\left\{C_{r}^{(m)}(z)\right\}$ are defined.

Proof. The above are special cases of more general results relating Padé quotients and convergents of corresponding continued fractions, established by Padé [3].

LEMma 6. Let $g(z)$ be the Stieltjes function of formula (5), and let the successive convergents $C_{r}(z)$ of its corresponding continued fraction have the form (9). Set $\tilde{C}_{r}(\lambda)=\lambda^{-1} C_{r}\left(\lambda^{-1}\right)$ for the functions $C_{r}(z)$ that are defined, so that, for such convergents,

$$
\tilde{C}_{2 r-1}(\lambda)=\frac{u_{1}}{\lambda+} \frac{u_{2}}{1+} \cdots \frac{u_{2 r-2}}{1+} \frac{u_{2 r-1}}{\lambda}, \quad \tilde{C}_{2 r}(\lambda)=\frac{u_{1}}{\lambda+} \frac{u_{2}}{1+} \cdots \frac{u_{2 r-1}}{\lambda+} \frac{u_{2 r}}{1} .
$$

Set $\tilde{C}_{0}(\lambda)=0$. Then, when $-\pi<\arg (\lambda)(\neq 0)<\pi$, the functions $\left\{\tilde{C}_{r}(\lambda)\right\}$ furnish a sequence of convex inclusion domains $\left\{\tilde{\mathfrak{L}}_{r}\right\}$ for the function

$$
G(\lambda)=\lambda^{-1} g\left(\lambda^{-1}\right)=\int_{a^{\prime}}^{b^{\prime}} \frac{d \tilde{\sigma}(s)}{\lambda+s} .
$$

$\mathfrak{\mathfrak { L }}_{r}$ is bounded by arcs of $\tilde{\mathfrak{T}}_{r}$ and $\tilde{\mathfrak{R}}_{r+1}$, successive members of a sequence of circles $\left\{\tilde{\mathfrak{R}}_{r}\right\}: \tilde{\mathfrak{R}}_{1}$ is the line joining the origin and the point $\tilde{C}_{1}(\lambda) ; \tilde{\mathfrak{R}}_{r}$ and $\tilde{\mathfrak{R}}_{r+1}$ intersect at the points $\widetilde{C}_{r-1}(\lambda)$ and $\widetilde{C}_{r}(\lambda)$, the tangents at the points of intersection forming an angle of $|\arg (\lambda)|$. When $\arg (\lambda)=0$, the values of the convergents $\left\{\widetilde{C}_{r}(\lambda)\right\}$ constitute an oscillating sequence of positive real numbers and provide a sequence of nested inclusion segments $\left\{\mathfrak{L}_{r}\right\}$ for the value of $G(\lambda): \tilde{\mathfrak{L}}_{2 r-1}$ is the segment $\left(\tilde{C}_{2 r-2}(\lambda), \tilde{C}_{2 r-1}(\lambda)\right], \mathfrak{\mathfrak { L }}_{2 r}$ is the segment $\left[\widetilde{C}_{2 r}(\lambda), \widetilde{C}_{2 r-1}(\lambda)\right)(r=1,2, \ldots)$.

For a fixed finite value of $r \geqq 1$ the domain $\mathfrak{\mathfrak { L }}_{r}$ of either type described above is an inclusion domain for the convergents $\boldsymbol{C}_{r^{\prime}}(\lambda)\left(r^{\prime}=r, r+1, \ldots\right)$.

Proof. The even order convergents $\left\{\tilde{C}_{2 r}(\lambda)\right\}$ may be expressed as

$$
\tilde{C}_{2 r}(\lambda)=\frac{v_{1}}{\lambda+w_{1}+} \frac{v_{2}}{\lambda+w_{2}+} \cdots \frac{v_{r}}{\lambda+w_{r}}
$$

they may be determined in this form by imposing the condition that the series expansion of $\tilde{C}_{2 r}(\lambda)$ in descending powers of $\lambda$ should agree with the series $\mathscr{F}(\lambda)$ $=\sum_{v=0}^{\infty} t_{v} \lambda^{-v-1}$ as far as the term $t_{2 r-1} \lambda^{-2 r}(r=1,2, \ldots)$. The continued fraction of which the functions $\left\{\tilde{C}_{2 r}(\lambda)\right\}$ are the successive convergents is said to be associated with the series $\mathscr{F}(\lambda)$. If $\mathscr{F}(\lambda)$ is generated by a function of the form

$$
F(\lambda)=\int_{a^{\prime \prime}}^{b^{\prime \prime}} \frac{d \tilde{\sigma}(s)}{\lambda+s}
$$


where $\left(a^{\prime \prime}, b^{\prime \prime}\right)$ is any segment of the real axis and $\sigma(s)$ is a bounded nondecreasing function for $a^{\prime \prime} \leqq s \leqq b^{\prime \prime}(G(\lambda)$ is, of course, such a function) and $\operatorname{Im}(\lambda) \neq 0$, the functions $\left\{\widetilde{C}_{2 r}(\lambda)\right\}$ furnish a sequence of nested circular inclusion domains $\left\{\tilde{\boldsymbol{\Theta}}_{2 r}\right\}$ for the value of $F(\lambda)$. $\tilde{\mathfrak{R}}_{0}$ is the real axis; $\tilde{\mathfrak{R}}_{2 r}$ lies inside $\tilde{\mathfrak{R}}_{2 r-2}$ touching the latter at $\tilde{C}_{2 r-2}(\lambda)(r=1,2, \ldots)$. (The existence of these circular inclusion domains was first discovered by Stieltjes [6]; they were used systematically by Hamburger [14] in his study of the moment problem for an infinite interval; their significance in the theory of functions of a complex variable was fully investigated by Nevanlinna [15].)

If $a^{\prime \prime} \geqq 0$ (and this is so for the function $G(\lambda)$ of formula (12)) the function

$$
F^{(1)}(\lambda)=\int_{a^{\prime \prime}}^{b^{\prime \prime}} \frac{s d \tilde{\sigma}(s)}{\lambda+s}
$$

is of the same type as $F(\lambda)$. The successive convergents $\left\{\widetilde{C}_{2 r}^{(1)}(\lambda)\right\}$ of its associated continued fraction generate a similar sequence of nested circular inclusion domains $\left\{\tilde{\Re}_{2 r}^{(1)}\right\}$ for $F^{(1)}(\lambda)$ : $\tilde{\Re}_{0}^{(1)}$ is the real axis; $\tilde{\Re}_{2 r}^{(1)}$ lies inside $\tilde{\Re}_{2 r-2}^{(1)}$, touching the latter at $\widetilde{C}_{2 r-2}^{(1)}(\lambda)(r=1,2, \ldots)$.

Since $F(\lambda)=t_{0} \lambda^{-1}-\lambda^{-1} F^{(1)}(\lambda)$, the latter inclusion domains yield a further system of circular inclusion domains $\left\{\tilde{\mathscr{\Re}}_{2 r+1}\right\}$ for $F(\lambda)$; each point of the circle $\tilde{\mathfrak{H}}_{2 r+1}$ is obtained from the symbolic equation

$$
\tilde{\Re}_{2 r+1}=t_{0} \lambda^{-1}-\lambda^{-1} \tilde{\Re}_{2 r}^{(1)} \quad(r=0,1, \ldots) .
$$

Furthermore, it follows from the conditions defining corresponding and associated continued fractions that

$$
\tilde{C}_{2 r+1}(\lambda)=t_{0} \lambda^{-1}-\lambda^{-1} \tilde{C}_{2 r}^{(1)}(\lambda) \quad(r=0,1, \ldots) .
$$

Hence $\tilde{\AA}_{1}$ is the line through the point $t_{0} \lambda^{-1}$ making an angle $-\arg (\lambda)$ with the positive real axis (i.e. the line joining the origin and $\tilde{C}_{1}(\lambda)$ ); $\tilde{\mathfrak{R}}_{2 r+1}$ lies inside $\tilde{\mathfrak{\Re}}_{2 r-1}$, touching the latter at $\widetilde{C}_{2 r-1}(\lambda)(r=1,2, \ldots)$.

It may easily be shown that $\tilde{\mathfrak{R}}_{2 r+1}$ intersects $\tilde{\mathfrak{K}}_{2 r}$ at $\tilde{C}_{2 r}(\lambda)$, and $\tilde{\mathfrak{R}}_{2 r+2}$ at $\tilde{C}_{2 r+1}(\lambda)$ $(r=0,1, \ldots)$, the tangents to the intersecting circles in both cases making an angle of $|\arg (\lambda)|$ at the points of intersection. If the circular domains $\tilde{\Re}_{r}$ and $\tilde{\Re}_{r+1}$ have been determined, that part of $\tilde{\mathfrak{R}}_{r}$ lying outside $\tilde{\mathfrak{K}}_{r+1}$ and that part of $\tilde{\mathfrak{K}}_{r+1}$ lying outside $\tilde{\mathfrak{R}}_{r}$ are both excluded from consideration as inclusion domains for $F(\lambda)$. What remains is the inclusion domain $\tilde{\mathfrak{L}}_{r}$.

When $\lambda$ tends to a finite positive real value and $\arg (\lambda)$ tends to zero, the convex inclusion domains $\left\{\tilde{\mathfrak{L}}_{r}\right\}$ tend to nested segments of the positive real axis, each containing the point $F(\lambda)$.

Since the domains $\left\{\tilde{\mathfrak{L}}_{r}\right\}$ form a nested sequence, $\tilde{\mathfrak{L}}_{r}$ is an inclusion domain for $\tilde{C}_{r^{\prime}}(\lambda)\left(r^{\prime}=r, r+1, \ldots\right)$.

An alternative derivation of the above theory was given by Henrici and Pfluger [16]. 
Lemma 7. Let $g(z)$ be the Stieltjes function of formula (5), denote the Padé quotients generated by $g(z)$ by $\left\{R_{i, j}(z)\right\}$, and let $m \geqq 0$ be a fixed finite integer. Then when $-\pi<\arg (z)(\neq 0)<\pi$ the values of distinct consecutive quotients (vide Lemma 1) of the sequence

$$
R_{0, m-1}(z), \quad R_{0, m}(z), \quad R_{1, m}(z), \quad R_{1, m+1}(z), \quad \ldots
$$

lie at the vertices of nested convex inclusion domains $\mathfrak{R}_{r}^{(m)}(r=1,2, \ldots)$ for the value of $g(z) . \mathfrak{R}_{r}^{(m)}$ is bounded by arcs of $\Re_{r}^{(m)}$ and $\mathfrak{\Re}_{r+1}^{(m)}$, consecutive members of a sequence of circles $\left\{\AA_{r}^{(m)}\right\}:{\Re_{1}^{(m)}}_{\text {is }}$ the line joining $R_{0, m-1}(z)$ and $R_{0, m}(z) ; \AA_{2 r}^{(m)}$ passes through

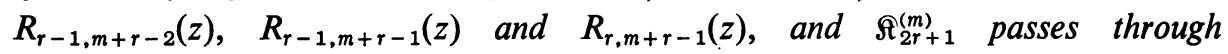
$R_{r-1, m+r-1}(z), R_{r, m+r-1}(z)$ and $R_{r, m+r}(z)(r=1,2, \ldots)$; at the points of intersection, the tangents to the intersecting circles make an angle of $|\arg (z)|$. When $z$ is real and positive, the values of the quotients (13) constitute an oscillating sequence of real numbers, and provide a sequence of nested inclusion segments $\left\{\mathcal{R}_{r}^{(m)}\right\}$ for the value of $g(z):$ if $m$ is even $\mathfrak{L}_{2 r-1}^{(m)}$ is the segment $\left[R_{r-1, m+r-2}(z), R_{r-1, m+r-1}(z)\right], \mathfrak{R}_{2 r}^{(m)}$ is the segment $\left[R_{r, m+r-1}(z), R_{r-1, m+r-1}(z)\right](r=1,2, \ldots)$; if $m$ is odd, the limits defining these segments must be reversed.

For a fixed finite value of $r \geqq 1, \Omega_{2 r-1}^{(m)}$ is an inclusion domain for the Padé quotients $R_{r+r^{\prime}-1, m+r+r^{\prime}-2}(z), R_{r+r^{\prime}-1, m+r+r^{\prime}-1}(z)$ and $\mathcal{L}_{2 r}^{(m)}$ is an inclusion domain for $R_{r+r^{\prime}-1, m+r+r^{\prime}-1}(z), R_{r+r^{\prime}, m+r+r^{\prime}-1}(z)\left(r^{\prime}=0,1, \ldots\right)$.

Proof. Substituting $\lambda=z^{-1}$ in the formulae of Lemma 6, we first obtain a sequence of nested inclusion domains $\left\{\tilde{\mathfrak{L}}_{r}^{(m)}\right\}$ for the function $z g^{(m)}(z)$, where $g^{(m)}(z)$ is defined by formula (10); the vertices of these inclusion domains occur at the points $\left\{z C_{r}^{(m)}(z)\right\}$ where $C_{r}^{(m)}(z)$ is as defined in Lemma 5. The inclusion domains $\left\{\mathcal{L}_{r}^{(m)}\right\}$ are obtained by use of the symbolic equations

$$
\mathfrak{Q}_{r}^{(m)}=\sum_{\nu=0}^{m-1} t_{v} z^{\nu}+z^{m-1} \mathfrak{L}_{r}^{(m)} \quad(r=1,2, \ldots) .
$$

The vertices of the inclusion domains $\mathfrak{L}_{r}^{(m)}$ occur at the points

$$
\sum_{\nu=0}^{m-1} t_{v} z^{v}+z^{m-1}\left\{z C_{r}^{(m)}(z)\right\}
$$

and these are, successively, the values of $R_{r, m+r-1}(z), R_{r, m+r}(z)$ given by formulae (11).

It has only to be remarked that the closed segment of the real axis joining the end points $\alpha, \beta$ is conventionally denoted by $[\alpha, \beta]$ if $\beta>\alpha$ and by $[\beta, \alpha]$ if $\beta<\alpha$.

Finally, we remark that when $g(z)$ is a rational function, only finitely many of the initial quotients of the sequence (13) are distinct, and (13) terminates with an infinite sequence of quotients identical with $g(z)$; the values of each of those identical quotients are, however, contained in $\mathfrak{R}_{r}^{(m)}$. 
For the sake of completeness we mention that, although we have no need of them, inclusion domains $\hat{\mathfrak{Q}}_{r}^{(m)}$ for $g(z)$ may also be determined from the quotients lying below the subprincipal diagonal of the Padé table generated by $g(z)$ : we first obtain a sequence of inclusion domains $\left\{\mathcal{R}_{r}^{\prime(m)}\right\}$ for the function $\hat{g}(z)$ defined by the formula

$$
g(z)=t_{0} /(1+z \hat{g}(z))
$$

and submit these to the symbolic transformation

$$
\hat{\mathfrak{Q}}_{r}^{(m)}=\frac{t_{0}}{\left(1+z \mathfrak{Q}_{r}^{(m)}\right)} \cdot
$$

However, it is possible that $\mathfrak{L}_{r}^{\prime(m)}$ includes the point $-z^{-1}$, and hence the $\left\{\hat{\mathfrak{L}}_{r}^{(m)}\right\}$ may not be finite regions.

Proof of the main theorem. We first consider those progressive sequences of the theorem that are restricted to lie in that part of the Padé table bounded from below by the subprincipal diagonal.

For nonnegative real values of $z$ belonging to the cut disc $\mathfrak{D}$, i.e. for $0 \leqq z<b^{-1}$, we know from Lemmata 1 and 4 that the values of $R_{r, r-1}(z)\left(R_{r, r}(z)\right)(r=0,1, \ldots)$ form a nondecreasing (nonincreasing) sequence of numbers converging to the value of $f(z)$.

The function $f(z)$ has simple poles at the points $\left\{-b_{v}^{-1}\right\}$ (where $-b_{n}^{-1}<\cdots$ $\left.<-b_{2}^{-1}<-b_{1}^{-1}\right)$ on the negative real axis, and further singularities confined to the segment of the negative real axis $\left(-a^{-1},-b^{-1}\right)$ (where $\left.-b^{-1}<-b_{n}^{-1}\right)$ the nature and distribution of which depend upon the structure of $\sigma(s)$. Lemma 3 can be applied to the Padé table generated by $f(z)$ and, in particular, when $0 \leqq z<b^{-1}$, the sequence $R_{n, n+r-1}(z)(r=0,1, \ldots)$ converges to $f(z)$.

For a fixed finite value of $r \geqq 0$, the backward diagonal sequences proceeding from $R_{n, n+2 r-1}(z)$ to $R_{n+r, n+r-1}(z)$ and from $R_{n, n+2 r}(z)$ to $R_{n+r, n+r}(z)$ are, when $0 \leqq z<b^{-1}$, nondecreasing and nonincreasing respectively (Lemma 1). Since the end points of these sequences themselves belong to sequences converging to $f(z)$, it follows that when $0 \leqq z<b^{-1}$ all progressive sequences of Padé quotients restricted as described above converge to $f(z)$.

Any point of $\mathfrak{D}$ not belonging to the negative real axis lies in $\mathfrak{D}^{\prime}$, the intersection of $\mathfrak{D}$ and the sector $-\pi+\delta \leqq \arg (z) \leqq \pi-\delta$, where $\delta$ is an arbitrarily small positive real number. The row sequence $R_{n, n+r-1}(z)(r=0,1, \ldots)$ converges uniformly to $f(z)$ in $\mathfrak{D}$ (Lemma 3 ) and, therefore, in $\mathfrak{D}^{\prime}$. Thus given any finite positive nonzero real number $\eta$, a finite positive integer $r^{\prime}$ can be found such that $\left|f(z)-R_{n, n+r}(z)\right| \leqq \eta$ for $r=r^{\prime}, r^{\prime}+1, \ldots$ and for all $z$ in $\mathfrak{D}^{\prime}$. Hence $\left|R_{n, n+r}(z)-R_{n, n+r+1}(z)\right| \leqq 2 \eta$ for $r=r^{\prime}, r^{\prime}+1, \ldots, R_{n, n+r}(z)$ and $R_{n, n+r+1}(z)$ are the vertices of an inclusion domain for $f(z)$ and all the quotients $R_{n+r^{\prime \prime}, n+r+r^{\prime \prime}}(z), R_{n+r^{\prime \prime}, n+r+r^{\prime \prime}+1}(z)\left(r^{\prime \prime}=0,1, \ldots\right)$ (Lemma 7). This inclusion domain is bounded by arcs of circles intersecting at $R_{n, r}(z)$ and $R_{n, r+1}(z)$, the tangents to which at the points of intersection form an 
angle of $|\arg (z)|$. A circle of radius $2 \eta / \sin \delta$ about $f(z)$, therefore, includes this inclusion domain. In short we have shown that

$$
\left|f(z)-R_{n+i, n+r^{\prime}+i+j}(z)\right| \leqq 2 \eta / \sin \delta \quad(i=0,1, \ldots ; j=0,1, \ldots)
$$

for all $z$ in $\mathfrak{D}^{\prime}$. Within and upon the boundary of $\mathfrak{D}^{\prime}, f(z)$ is analytic. Thus, if $M^{\prime}$ is the maximum modulus of $f(z)$ upon the boundary of $\mathfrak{D}^{\prime}$, and we set $M^{\prime \prime}=M^{\prime}$ $+2 \eta / \sin \delta$,

$$
\left|R_{n+i, n+r^{\prime}+i+j}(z)\right| \leqq M^{\prime \prime} \quad(i=0,1, \ldots ; j=0,1, \ldots)
$$

uniformly for all $z$ in $\mathfrak{D}^{\prime}$.

We must now consider the points of $\mathscr{D}$ not belonging to $\mathfrak{D}^{\prime}$, i.e. those points of $\mathscr{D}$ lying in the neighborhood of the negative real axis. Such points satisfy the condition $|z| \leqq b_{1}^{-1}-\delta^{\prime}$, where $\delta^{\prime}$ is a fixed arbitrarily small positive real number. From Lemmata 4 and 5 , the quotients $R_{r, m+r-1}(z)(r=n, n+1, \ldots ; m=0,1, \ldots)$ have a representation of the form

$$
R_{r, m+r-1}(z)=\sum_{\nu=0}^{m-1} t_{v} z^{v}+z^{m} \sum_{v=1}^{r} \frac{\mu_{r, v}^{(m)}}{1+\beta_{r, v}^{(m)} z}
$$

where $(-1)^{m} \mu_{r, v}^{(m)}>0, a<\beta_{r, v}^{(m)}<b(\nu=1,2, \ldots, r)$ and $\sum_{v=1}^{r} \mu_{r, v}^{(m)}=t_{m}$, the series $f(z)$ of formula (3) being the expansion in ascending powers of $z$ of the function $f(z)$. Since $f(z)$ converges for $|z| \leqq b_{1}^{-1}-\frac{1}{2} \delta^{\prime}$, we have $0 \leqq\left|\sum_{v=0}^{m-1} t_{v} z^{v}\right| \leqq M_{2}, 0 \leqq\left|t_{m} z^{m}\right| \leqq M_{3}$ $(m=0,1, \ldots)$ for all such values of $z, M_{2}$ and $M_{3}$ being two finite constants. Furthermore, when $|z| \leqq b_{1}^{-1}-\delta^{\prime}$, a positive real number $\delta^{\prime \prime}$ can be found such that $\left|1+\beta_{r, \nu}^{(m)} z\right| \geqq \delta^{\prime \prime}(r=n, n+1, \ldots ; \nu=1,2, \ldots, r ; m=0,1, \ldots)$. In short, there exists a finite positive real number $M^{m}$ such that an inequality of the form (14) with $M^{\prime \prime}$ replaced by $M^{m}$ holds uniformly for $|z| \leqq b_{1}^{-1}-\delta^{\prime}$.

Setting $M=\max \left(M^{\prime \prime}, M^{\prime \prime}\right)$, it follows that an inequality of the form (14) with $M^{\prime \prime}$ replaced by $M$ holds uniformly for $z$ in $\mathfrak{D}$. We have shown that the quotients of any progressive sequence of the Padé table generated by $f(z)$, formed from the quotients lying on or above the forward diagonal sequence $R_{n+r, n+r+r^{\prime}}(z)(r=0$, $1, \ldots)$ and on or below the row sequence $R_{n, n+r+r}(z)(r=0,1, \ldots)$, are uniformly bounded in modulus for all $z$ in $\mathfrak{D}$. Since the quotients of such a sequence converge to $f(z)$ for all nonnegative real values of $z$ in $\mathfrak{D}$, it follows from the Stieltjes-Vitali theorem that this sequence of quotients converges uniformly to $f(z)$ in $\mathfrak{D}$.

For $r^{\prime \prime}=0,1, \ldots, r^{\prime}$ all diagonal sequences $R_{n+r, n+r^{\prime \prime}+r-1}(z)(r=0,1, \ldots)$ converge uniformly to $f(z)$ in $\mathfrak{D}$ (Lemma 4). Hence all progressive sequences restricted as described at the commencement of the proof converge uniformly to $f(z)$ in $\mathfrak{D}$.

The above analysis can be extended to the Padé quotients $\left\{\hat{R}_{i, j}(z)\right\}$ generated by the function $\hat{f}(z)$ related to $f(z)$ by formula (7) and having the representation (8). It follows from Lemma 2 that we have proved the theorem in its entirety.

In conclusion we remark that the theorem of this paper contains by implication a number of subsidiary results obtained by replacing $n$ by a lesser integer and incorporating one or more of the terms $\left\{M_{v} /\left(1+b_{v} z\right)\right\}$ into the integral expression of 
formula (1) by extending the range of integration and suitably modifying the function $\sigma(s)$. Again, depending upon the nature of $\sigma(s)$, the theorem is itself capable of extension.

\section{REFERENCES}

1. O. Perron, Die Lehre von den Kettenbrüchen, Dritte, Verbesserte und erweiterte Aufl. Bd. II, Analystich-funktionentheoretische Kettenbrüche, Teubner Verlagsgesellschaft, Stuttgart, 1957. MR 19, 25.

2. H. S. Wall, Analytic theory of continued fractions, Van Nostrand, Princeton, N. J., 1948. MR 10, 32.

3. H. Padé, Sur la représentation approchée d'une fonction par des fractions rationelles, Ann. Sci. École Norm. Sup. 9 (1892), (supplement), 1-93.

4. C. G. J. Jacobi, Uber die Darstellung einer Reihe gegebner Werthe durch einer gebrochnen rationale Funktion, J. Reine Angew. Math. 30 (1845), 127-156.

5. G. Frobenius, Uber Relationen zwischen den Näherungsbruchen von Potenzreihen, J. Reine Angew. Math. 90 (1881), 1-17.

6. T. J. Stieltjes, Recherches sur les fractions continues, Ann. Fac. Sci. Univ. Toulouse 8 (1894), 1-22; ibid. 9 (1895), 1-47.

7. E. B. Van Vleck, On an extension of the 1894 memoir of Stieltjes, Trans. Amer. Math. Soc. 4 (1903), 297-332.

8. H. S. Wall, On the Padé approximants associated with the continued fraction and series of Stieltjes, Trans. Amer. Math. Soc. 31 (1929), 91-116.

9. P. Wynn, Upon the Padé table derived from a Stieltjes series, SIAM J. Numer. Anal. $5(1968)$, 805-834. MR 39 \#1091.

10. R. de Montessus de Ballore, Sur les fractions continues algébriques, Bull. Soc. Math. France 30 (1902), 28-36.

11. J. Hadamard, Essai sur l'étude des fonctions données par leur developpement de Taylor, J. Math. 8 (1892), 101-186.

12. P. Tschebyscheff, Sur le developpement des fonctions á une seule variable, Bull. Acad. Imp. Sci. St. Petersburg 1 (1860).

13. A. Markoff, Deux demonstrations de la convergence de certaines fractions continues, Acta Math. 19 (1895), 93-104.

14. H. Hamburger, Ueber eine Erweiterung des Stieltjes'schen Momentenproblems, Math. Ann. 81 (1920), 235-319; ibid. 82 (1921), 120-164, 168-187.

15. R. Nevanlinna, Asymptotische Entwickelungen beschränkter Funktionen und das Stieltjes'sche Momentenproblem, Ann. Acad. Sci. Fenn. Ser. A I 18 (5) (1922).

16. P. Henrici and P. Pfluger, Truncation error estimates for Stieltjes fractions, Numer. Math. 9 (1966), 120-138. MR 35 \#3856.

Department of Mathematics, Louisiana State University in New Orleans, New OrLeans, Louisiana 70122 